\title{
La inteligencia emocional: una estrategia para mejorar el rendimiento académico, la comunicación y la pedagogía crítica.
}

\section{The emotional intelligence: a strategy to improve academic performance, communication and critical pedagogy.}

Mgs. Nelly Noemí Andrade Albán. ${ }^{1}$, Dr. Mcs. Jorge Rodrigo Andrade Albán. ${ }^{2}$, Md.Esp. Noemí Rocío Andrade Albán. ${ }^{3}$, Dr, PhD. Víctor Peñafiel Gaibor. ${ }^{4} \&$ Mcs.Angel Leonardo Andrade. ${ }^{5}$

Recibido: 01-09-2017 / Revisado 01-11-2017 Aceptado: 11-12-2017/ Publicado: 01-01-2018

\begin{abstract}
.
DOI: https://doi.org/10.33262/cienciadigital.v2i1.25

The present research called Emotional intelligence: a strategy to improve academic performance, communication and critical pedagogy, aims to investigate the incidence of emotional intelligence in the academic performance of students in the tenth year of General Basic Education of the Unit Educational "Rumiñahui", canton Ambato. The study carried out has relevance when investigating the emotional intelligence, through the theories and competences, sustaining in the multiple intelligences to motivate the scholar in the learning, the development of skills, the solution of problems from the logical, dynamic and progressive vision; contributes to the improvement of academic performance, by backing on critical pedagogy; . The methodology was carried out an analysis from the qualitative and quantitative perspective; the modality of field, facilitated the reflection on the scholastic performance; the documentary bibliographic study contributed in the knowledge, the cognitive development through documents and investigations carried out previously; exploratory, descriptive and correlational levels favored in the collection, selection and systematization of information; when the survey was used as a means of collection, and the structured questionnaire applied to a population of eighty-five students and twelve basic education teachers.

Keywords: Affectivity, Learning, Teaching, Multiple Intelligences, Academic Performance.

\footnotetext{
${ }^{1}$ Distrito Educativo Intercultural Bilingue, Ambato, Ecuador , nellycym@ hotmail.com ${ }^{2}$ Universidad Técnica de Ambato, Facultad de Ciencias Humanas y de la Educación, jr.andrade @uta.edu.ec ${ }^{3}$ Universidad Técnica de Ambato, Facultad de Ciencias de la Salud, nr.andrade@uta.edu.ec ${ }^{4}$ Universidad Técnica de Ambato, Facultad de Ciencias de la Salud, vi.penafiel@uta.edu.ec ${ }^{5}$ Universidad Técnica de Ambato, Facultad de Ciencias de la Salud, aandrade@pronaca.com
} 


\section{Resumen.}

La presente investigación denominada La inteligencia emocional: una estrategia para mejorar el rendimiento académico, la comunicación y la pedagogía crítica, tiene por objetivo investigar la incidencia de la inteligencia emocional en el rendimiento académico de los estudiantes de décimo año de Educación General Básica de la Unidad Educativa "Rumiñahui", cantón Ambato. El estudio efectuado tiene relevancia al investigar la inteligencia emocional, a través de las teorías y competencias, sustentándose en las inteligencias múltiples para motivar al escolar en el aprendizaje, el desarrollo de destrezas, la solución de problemas desde la visión lógica, dinámica y progresiva; aporta en el mejoramiento del rendimiento académico, al respaldarse en la pedagogía crítica; . La metodología se efectúo un análisis desde la perspectiva cualitativa y cuantitativa; la modalidad de campo, facilitó la reflexión sobre el desempeño escolar; el estudio bibliográfico documental aportó en el conocimiento, el desarrollo cognitivo a través de documentos e investigaciones efectuadas con anterioridad; los niveles exploratorios, descriptivo y correlacional favorecieron en la recolección, selección y sistematización de la información; al utilizarse como medio de recolección la encuesta, y el cuestionario estructurado aplicado a una población de ochenta y cinco educandos y doce docentes de educación básica.

Palabras Claves: Afectividad, aprendizaje, enseñanza, inteligencias múltiples, rendimiento académico.

\section{Introducción.}

Iniciando desde la importancia del fortalecimiento de la inteligencia emocional para el desarrollo integral; (López E. , La educación emocional en el aprendizaje infantil, 2015) en el trabajo efectuado con la temática educacion emocional en el aprendizaje, expone que; la inteligencia emocional favorece en el desempeño académico, integra el autoconocimiento, autocontrol, automotivación, el reconocimiento de sentimientos positivos o negativos y las consecuencias que producen; componentes que aportan en la confianza y seguridad en la adquisición de capacidades y la toma de decisiones.

Según el estudio establecido por (Martineaud \& Engelhart, 2014) el autocontrol favorece en el comportamiento responsable que disminuye las conductas inadecuadas a través de la conciencia y la reflexión; mientras, (Navarro, Rodríguez, \& Eirín, 2016) exterioriza que la automotivación favorece en la motivación para alcanzar aspiraciones, al generar fortaleza en el logro de finalidades y vencer obstáculos; (Pérez L. , 2013) agrega que aumenta el optimismo y la iniciativa en el educando para la consecución de tareas, el trabajo en equipo y relaciones interpersonales. (Pérez L. , 2013), manifiesta que la empatía es una capacidad que aporta para conocer las emociones de las demás personas, favorece en la capacidad 
cognitiva, la confianza y la expresión de pensamientos; por esta razón, (Hernández, 2015) deduce que la empatía, beneficia en la adaptación emocional y en la sociabilidad; el educando que ha fomentado la empatía, se siente comprendido, confía en las personas y demuestra seguridad sus actividades.

Las habilidades sociales, se centran en el liderazgo para direccionar, gestionar crear e innovar el conocimiento en el grupo de personas a través de la participación y valoración personal; por las razones mencionadas los objetivos del trabajo fueron: investigar, analizar la incidencia de la inteligencia emocional en el rendimiento académico; determinar las causas que afectan en el rendimiento académico y analizar algunos lineamientos respecto a la inteligencia emocional y su aplicación en el rendimiento académico de los estudiantes de décimo año, de Educación General Básica de la Unidad Educativa "Rumiñahui", cantón Ambato".

\section{Inteligencia emocional en el fortalecimiento de las competencias.}

En el ambiente educativo, las competencias emocionales y habilidades sociales deben ser aplicadas a la vivencia real de los estudiantes, encausándose dentro de lineamientos que posee el currículo y que debe desarrollar el profesorado, siendo que el aprendizaje de las habilidades se relacionan con la practica en el ambiente educativo, mediante la comunicación y el diálogo; elementos principales para trabajar las habilidades emocionales, comportamientos y valores.

Para el autor (López P. , 2015), la inteligencia emocional se relaciona con la conciencia, comprensión, conocimiento y relaciones interpersonales, indispensable para disminuir impulsos, agresiones, conflictos, al obtener como beneficio mejores decisiones ante sentimientos de ira, miedo, que influyen en el estado de ánimo; mientras los estudios de (Álvarez, 2017) se enfocan en la importancia del manejo de emociones que ayuda en el control del estrés, comportamientos inapropiados, incertidumbre y ansiedad; además, persiste en el desarrollo de las habilidades sociales, el autoconocimiento, la interacción, parte de las competencias emocionales.

Según los autores: (Aparicio, González, Portal, García, \& Lorente, 2016) al fortalecer la inteligencia emocional el educando; aporta para el desarrollo de la capacidad para motivarse, superar dificultades, es capaz de reconocer emociones como el miedo, la irritación, desconsuelo, satisfacción, el razonamiento; apoya en la conciencia para realizar tareas, factores que promueven el éxito y bienestar; ante el criterio emitido por los autores, (Jiménez, 2016) en el estudio de las inteligencias emocionales, deduce que el niño al hablar de sus miedos, demostrar alegría en sus diferentes manifestaciones: grito, riza, abrazos, palmadas, demuestra seguridad, y autodisciplina; (Silva, 2013) complementa que la 
inteligencia se relaciona con el coeficiente intelectual, el autoconocimiento y la toma de decisiones favorecen en la evaluación de valores; deduciéndose que perfeccionar la inteligencia de las emociones es acrecentar la productividad del conocimiento, mantener relaciones interpersonales y el equilibrio emocional.

Para los autores; (Aguaded \& Pantoja, 2015) El desarrollo personal favorece en el control de emociones, aporta la competencia del liderazgo, facilita la intuición de los sentimientos de los demás; (Franco, 2014) incluye que el alcance de metas requiere del dominio emocional que influye en la motivación, el conocimiento, la confianza, el rendimiento, impulsa en las relaciones interpersonales, la responsabilidad y el auto concepto; mientras (Buitrago, 2012), sintetiza que la inteligencia emocional es adquirida por el ser humano durante los primeros años de vida, los padres a través de afectividad, desarrollan en el infante confianza y seguridad; además, el niño desarrolla en el ambiente experiencias de aprendizaje que favorecen en el progreso cognitivo.

La educación emocional es importante para el aprendizaje de los educandos, porque influyen en el impulso de competencias, el conocimiento y el respeto; (Cantó \& Solbes, 2016)al compartir su opinión demuestra fortaleza ante las dificultades, acepta sus limitaciones y establece relaciones con otras personas, primordiales para el desarrollo, los vínculos y la identidad.

La inteligencia emocional se relaciona con las habilidades sociales que aportan para alcanzar el dominio en diferentes áreas conocimiento, el autor (Aguaded \& Pantoja, 2015) menciona que intervienen en este proceso la comunicación, para disminuir los conflictos establece una mediación y solucionar problemas

La autoconfianza es alcanzada a través del manejo de emociones, la construcción de vehículos sociales la integración hacia los equipo de trabajo mediante netas compartidos que orientan en la visión compartida, por lo expuesto los investigadores(García \& Giménez, 2010) incorporan que el autocontrol integra el manejo de emociones sean positivas o negativas para mantener un equilibrio en la expresión, evitando la creación de conflictos por impulsos no controlados (Hose, 2016) en su investigación enfatiza en el control de emociones y menciona que el automanejo de emociones integra la honestidad, lealtad y creatividad que forman parte de la fiabilidad, mientras la conciencia integra a la responsabilidad el cumplimiento, organización, la adaptabilidad, el autor (López E. , 2005) expone que la inteligencia emocional fomenta la sensibilidad y flexibilidad para vencer obstáculos y resolver situaciones conflictivas; el logro de orientación enlaza la dirección y la excelencia, siendo que la iniciativa aporta para la actuación frente a cualquier dificultad .

En el desarrollo de habilidades efectivas y reflexivas, la inteligencia se relaciona al cumplir pautas y normas fundamentales para la convivencia, es así como (Moreno, 2016), hace 
referencia a las áreas de aprendizaje, el conocimiento de sí mismo que apoya para la autonomía personal; se establece que mediante la identificación de necesidades y pensamientos, se fomenta el dominio de emociones y la expresión en forma libre manteniendo el respeto hacia las otras personas.

El autor, (Mercadé, 2016) en la investigación vinculada con los tipos de inteligencia da a conocer que para alcanzar la inteligencia emocional, se efectúan actividades y programas que contienen dinámicas de integración, juegos para el autoconocimiento videos enfocados en la motivación, liderazgo, formación de grupos, relaciones interpersonales entre otros, los talleres tienen como misión fortalecer lazos de amistad.

En el ambiente psicopedagógico los educandos demuestran conductas inadecuadas entre los compañeros, demostrando actitudes agresivas, comportamientos violentos, lenguaje impropio, acciones que incrementan las tenciones, aumentan la violencia, disminuyen las relaciones positivas.

El compromiso del docente se centra en la creación de alternativas que aporten en la creación de alternativas para la convivencia, reflexión, el reconocimiento, la armonía y el establecimiento de relaciones sociales afectivas; siendo esencial la utilización de técnicas que apoyen para mejorar las capacidades de negociación y mediación.

\section{Las emociones en la comunicación y pedagogía crítica.}

Según el investigador (Fuentes, 1986), la inteligencia al colaborar para el progreso del nivel cognitivo se constituye en la base para el reconocimiento, la identificación y distinción de emociones que incrementa el autoconocimiento y estimula la valoración personal; (Murillo, 2006) despliega que durante los últimos tiempos a través de diversas actividades que el docente ha establecido para estimular la inteligencia emocional, influye en las capacidades intelectuales y en el rendimiento académico.

Los autores; (Harris \& Bennett, 2016) en el trabajo relacionada con la eficacia escolar, publican: en las relaciones sociales la inteligencia facilita la construcción de habilidades comunicativas para compartir sentimientos emociones, componentes importantes que estimulan las relaciones interpersonales, del mismo modo (Beltrán, 2016), despliega que la solidaridad a través del apoyo a los demás, la confianza para compartir experiencias aportan para la construcción de una imagen de sí mismo; y (Buitrago \& Herrera, 2014) reflexiona que la confianza en sí mismo favorece en el bienestar, al disminuir la sensación de soledad, abandono, tristeza que influyen en la baja autoestima.

Para; (Touron, 2014) en el ámbito educativo la inteligencia emocional, mejora el aprendizaje integral, se centraliza en el autoconocimiento para evaluar el rendimiento escolar, fomenta la autoestima, el control emocional que conlleva al mejoramiento de 
actitudes para expresión; mientras (Castro, 2014)en su estudio relacionado con la inteligencia emocional expone que el control de emociones disminuye problemas de ansiedad y depresión, al favorecer en actitudes positivas, la empatía, el rendimiento académico de esta manera la inteligencia emocional aporta en el aprendizaje, el conocimiento y las habilidades en los aspectos cognitivos, la afectividad y la sociabilidad.

Los estudiosos; (Valle, Cabanach, Rodríguez, Núñez, \& González, 2016), analizan en las emociones favorecen en la motivación, el entusiasmo, la afectividad, satisfacción, mientras la carente autoestima es generada por la desconfianza, resentimiento, intimidación, prejuicio, decepción, entre otros factores; el autor (Bassedas, 2015) profundiza que el autoestima y autoconocimiento en el ambiente educativo integra la inteligencia emocional, autoconciencia, el pensamiento creativo, la habilidad mental, comunicación, expectativas y la forma de enseñar.

\section{Metodología.}

La investigación se enfocó en la investigación de la inteligencia emocional, integra como componentes la atención entendimiento, reflexión para alcanzar el autoconocimiento y lograr la autorrealización, además se determinó y analizó las causas que condicionan el rendimiento académico, en busca de mejorar las prácticas formativas para disminuir las agresiones físicas, psicológicas y verbales, contratándose en los procesos de liderazgo, el trabajo educativo, la responsabilidad en las tareas y el desempeño académico. La investigación afectada bajo la temática: inteligencia emocional y el rendimiento académico se caracteriza por la aplicación de un enfoque cualitativo a través de análisis y síntesis indaga en las característica $\mathrm{y}$, principios de carácter científico desde una perspectiva general; mientras la utilización del enfoque cuantitativo al tener como característica el planteamiento del problema; la obtención de información, comprobación de la hipótesis a través de un análisis estadístico que describe a las variables para evitar perjuicios y aspectos personales.

La modalidades de estudio fueron seleccionadas de acuerdo a la complejidad de la investigación; mencionándose las siguientes; la modalidad bibliográfica favoreció en el estudio de la inteligencia emocional, el rendimiento académico, el pensamiento crítico, la comunicación a través del manejo y adquisición de información con carácter científico que respaldó la comparación de estudios teóricos para conocer la realidad, la evaluación y la vida social, al organizar, examinar y construir reflexiones, para obtener interpretaciones desde la interacción, la orientación, el descubrimiento y síntesis de la temática de estudio.

A través de la práctica descriptiva se examinó las particularidades la problemática del carente desarrollo de la inteligencia emocional y el rendimiento académico; que condujo a 
definir y formular la hipótesis a través de la encuesta que facilitó la recolección de datos, el establecimiento de semejanzas, diferencias e interacciones esenciales, mientras la investigación exploratoria orientó en la identificación de las consecuencias producidas por el problema; para plantear la hipótesis, determinar las preguntas, para familiarizar al investigador con el estudio de la inteligencia emocional y el rendimiento académico.

La investigación explicativa, permitió la descripción de teorías, conceptos, generalizaciones que aportaron para determinar relaciones entre las causas y efectos; el estudio correlacional analizó la inteligencia emocional y el rendimiento académico, mediante datos obtenidos en la aplicación de la encuesta, aproximando al descubrimiento de relaciones y la valoración de semejanzas para la formulación de la hipótesis y su aprobación a través de instrumentos estadísticos. Para la ejecución de la investigación se organizó un grupo de ochenta y cinco educandos y 12 docentes que laboran en la Unidad Educativa Rumiñahui; detallándose a continuación:

Tabla $\mathbf{N}^{\mathbf{0}}$ 1. Población.

\begin{tabular}{lll}
\hline OPCIONES & FRECUENCIA & PORCENTAJE \\
\hline Estudiantes & 85 & $96 \%$ \\
Docentes & 12 & $4 \%$ \\
Total & $\mathbf{9 7}$ & $\mathbf{1 0 0 \%}$ \\
\hline
\end{tabular}

Elaborado por: Guzmán Núñez Blanca Maritza.

En la muestra se seleccionó a la totalidad de la población, desde una perspectiva no probabilística al buscar profundizar en la problemática mediante la aportación de los participantes, para entender, comprender, reflexionar y dar respuestas a interrogantes planteadas en la investigación, la información cuantitativa con fines estadísticos se basó en procesos informáticos, se cumplió con la redacción de interrogantes, utilizando un lenguaje comprensible para evitar dificultades en la aplicación y facilitar la codificación, el análisis e interpretación de datos, fue aplicada a ochenta y cinco educandos y doce docentes de Educación Básica; mientras el cuestionario estructurado, facilitó la recopilación de información al concentrarse es las variables de estudio; las interrogantes aplicadas fueron en un número de trece; se utilizó alternativas cerradas y numéricas, en la redacción mantuvieron la precisión, la formalidad neutral; caracterizándose por la facilidad en la elección de respuestas. 


\section{Resultados.}

Para el análisis e interpretación de resultados se adoptó por la elección de diez ítems; se incorporó las concernientes tablas y gráficos que dieron notabilidad en la investigación, consiguiéndose los siguientes resultados:

Para el análisis e interpretación de resultados se adoptó por la elección de diez ítems; se incorporó las concernientes tablas y gráficos que dieron notabilidad en la investigación, consiguiéndose los siguientes resultados:

Ítem N. 2. ¿Usted demuestra autoconfianza y seguridad; alcanza el éxito personal y académico?

Tabla 2. Autoconfianza y optimismo.

\begin{tabular}{lll}
\hline OPCIONES & FRECUENCIA & PORCENTAJE \\
\hline $\mathrm{Si}$ & 49 & $58 \%$ \\
$\mathrm{No}$ & 36 & $42 \%$ \\
Total & $\mathbf{8 5}$ & $\mathbf{1 0 0 \%}$ \\
\hline
\end{tabular}

Fuente: Guía de preguntas aplicada en la investigación.

Elaborado por: Guzmán Núñez Blanca Maritza.

El 58\% si demuestra autoconfianza y seguridad, alcanza el éxito personal y académico, mantiene una adecuada autoestima y autoconfianza, se acepta como es, reconoce sus limitaciones y cualidades, busca la superación personal, mientras $42 \%$ no alcanza el éxito personal y académico, no aprende de sus experiencias, no potencia las virtudes, barreras que afectan en el trabajo para alcanzar la superación. Los estudiantes demuestran autoconfianza y seguridad, plantea metas personales y afronta retos; deduciéndose que el docente durante el cumplimiento de sus funciones en la labor pedagógica aportan para el bienestar emocional y cognitivo del educando; la carente confianza y responsabilidad del escolar condiciona la comprensión, el bienestar personal y el rendimiento académico, al mantener dificultades para reconocer sus valores crenado barreras para el desarrollo de su identidad. Mientras el carente planteamiento de expectativas realistas, desfavorece en el desarrollo de la autoconfianza para alcanzar el éxito y el desarrollo personal; se obtiene que el docentes en la práctica educativa a través de actividades motivacionales fomenta el desarrollo de la inteligencia emocional, la cooperación y solidaridad para mantener un clima de aprendizaje adecuado que beneficie en la adquisición de conocimientos que conllevan a expresar las ideas con libertad, autonomía y asertividad. 
Ítem N. 10: ¿Usted comprende, expresa experiencias, situaciones, saberes con pensamiento crítico, emite juicios significativos?

Tabla 3. Comprende, expresa experiencias, situaciones y saberes.

\begin{tabular}{lll}
\hline OPCIONES & FRECUENCI & PORCENTAJE \\
& $\mathrm{A}$ & \\
\hline $\mathrm{Si}$ & 41 & $38 \%$ \\
No & 54 & $62 \%$ \\
Total & 85 & $100 \%$ \\
\hline
\end{tabular}

Fuente: Guía de preguntas aplicada en la investigación.

Elaborado por: Guzmán Núñez Blanca Maritza.

El 38\% mediante actividades integradoras ha desarrollado la habilidad social, comprende, expresa experiencias, demuestra un pensamiento crítico y emite juicios significativos que potencializan e impulsan el aprendizaje, las capacidades cognitivas, el acceso y progresión en otras áreas del saber; mientras el $62 \%$ no cumple tareas, no participa en actividades grupales, aumentando la confusión en la adquisición de saberes, siendo carente el trabajo, compromiso, iniciativa y tenacidad para cumplir metas, es escasa la iniciativa y decisión exterioriza culpabilidad y resentimiento; lamentablemente los escolares no manejan conflictos, aumentando la agresividad, incomprensión e indisciplina, elementos condicionantes para el establecimiento de negociaciones para resolver problemas ni enfrentar dificultades. La acumulación de pensamientos, emociones y experiencias negativas, como positivas, las habilidades en los diferentes niveles aprendidos, promueven la seguridad y la confianza en cada escolar.

Figura. 1. Histograma de las curvas de análisis de las interrogantes.

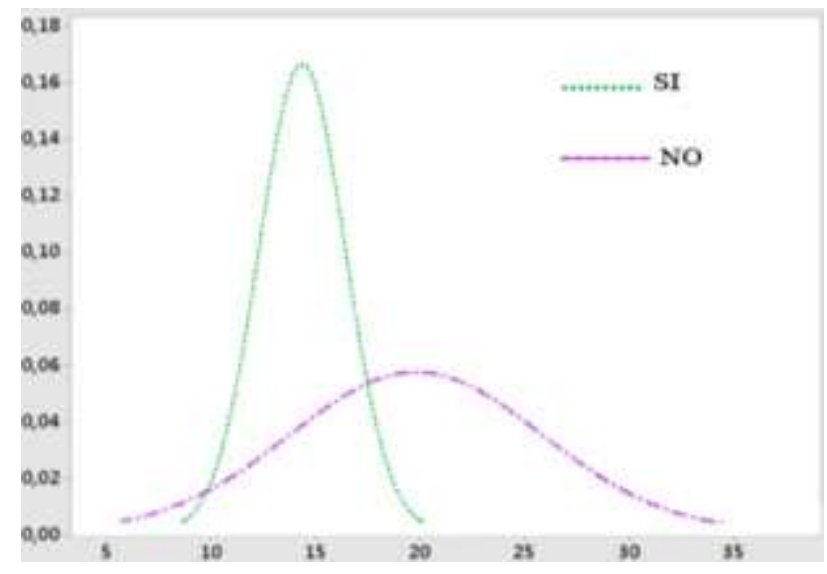

Elaborado por: Grupo de Investigación. 
En el histograma de la figura 1; se visualiza las curvas de análisis obtenidos de las interrogantes seleccionadas, verificándose que sobre la mediana el estudiante demuestra autoconfianza y seguridad, alcanza el éxito personal y académico; sin embargo por debajo de la media se deduce que el educando posee dificultades en la comprensión, factor que condiciona el desarrollo d la inteligencia emocional y el rendimiento académico.

El carente reconocimiento de emociones, perjudica en la responsabilidad, la integración, la práctica de valores como la tolerancia y solidaridad; mientras que la aplicación de habilidades sociales generan confianza y seguridad para emitir criterios, pensamientos y emociones; sin embargo el escaso conocimiento de emociones propias afecta en el razonamiento e introversión, elementos que limitan el progreso en la discip0lina, la observación, investigación, curiosidad y el conocimiento.

El incumplimiento de metas, deteriora la construcción de conceptos y definiciones basadas en el razonamiento lógico; lo que condiciona la comprensión; la irresponsabilidad en la integración de equipos de trabajo, conlleva a la desconformidad, y baja autoestima; el educando responsable se adapta a cambios, coopera en el grupo, trabaja por el bien común, es asertivo, creador e innovador.

Los docentes en su mayoría manifiestan que los educandos a veces realizan actividades para el desarrollo de la comprensión, factor limitante para el conocimiento, raciocinio e introversión; menor porcentaje expone que siempre el escolar a través de actividades en el ambiente educativo desarrolla habilidades que favorecen en el estudio, abstracción, y análisis crítico.

Los maestros a través de diferentes actividades fomentan la práctica de valores que aporta en la cooperación, construcción del pensamiento crítico, exteriorización de actitudes positivas y habilidades sociales que fortalecen la relación entre compañeros; el escolar al manifestar baja autoestima, carente relación con los compañeros, aislamiento, agresividad e incomprensión, no desarrollan la concentración, abstracción, argumentación, la inferencia y deducción, deteriorando el rendimiento académico;

\section{Discusión.}

El carente reconocimiento de emociones propias y las conductas de los demás dificulta el manejo de sentimientos y afectos, lo que dificulta para mantener el equilibrio en sus decisiones; (Martín, 2013)un apropiado control de emociones influyen de manera positiva en el potencial reflexivo y la atención, que favorece en el rendimiento escolar, la autoestima y el autoconcepto; (Egido, 2016) el educando inicia con la construcción de habilidades como la expresión, evaluación, y el compartir emociones e inquietudes con los demás. 
La limitada confianza en sí mismo condiciona la perseverancia para el cumplimiento de proyectos, perjudica en el enfrentamiento de desafíos, crea barreras en la solución de conflictos y el rendimiento académico factor limitante para el clima escolar, la motivación y el trabajo en el aula; (Bernal, 2013) afortunadamente cuando el estudiante supera dificultades y obstáculos que se presentan en el diario vivir; (González, Torres, \& Carreres, 2017) fortalece las relaciones sociales, el desarrollo cognitivo y el aprendizaje, demuestra seguridad para alcanzar el éxito personal y académico.

El docente en la labor pedagógica a través de la innovación y actualización de conocimientos, ha logrado que el estudiante alcance el bienestar emocional, la autonomía y asertividad, al demostrar iniciativa, compromiso y competencia para cumplir tareas escolares; el autor (Silva F. , 2014) menciona: los estudiantes que exteriorizan desinterés en la enseñanza y el aprendizaje, manifiestan dificultades para cumplir tareas escolares, lo que incrementa la decepción, bajo rendimiento y abandono académico.

Los educandos que no comprenden, mantienen limitaciones en la expresión de experiencias para potenciar e impulsar el aprendizaje; lo que limita en la progresión en otras áreas del saber, en el razonamiento, habilidades y actitudes al influir de forma negativa en el trabajo autónomo, la cooperación y el desempeño escolar; según (Tapia, 2014), los escolares que han desarrollado la comprensión, argumentación, y síntesis durante el proceso enseñanza aprendizaje, tienen capacidad para emitir juicios valorativos, demuestra autonomía e independencia en la realización de actividades.

\section{Conclusión.}

La inteligencia emocional influye para mejorar el rendimiento académico, la capacidad para reconocer las emociones propias y las conductas de los demás, al favorecer un el reconocimiento de emociones, el control de la ansiedad, angustia, e impaciencia; para el manejo adecuado de las relaciones interpersonales., que aportan en la autoconfianza y seguridad para el bienestar emocional y cognitivo

A través de la Inteligencia emocional es importante fortalecer la autoconfianza para alcanzar el desarrollo personal, mejorar el rendimiento académico, la libertad, autonomía y asertividad, al incrementar .la perseverancia, superación de dificultades y obstáculos que se presentan en el diario vivir, al demostrar iniciativa, responsabilidad y compromiso para el progreso en el aprendizaje.

Fortalecer los componentes de la inteligencia emocional como; la autoestima, autovaloración, el autoconocimiento para superar desafíos, alcanzar la identidad propia, trabajo, compromiso e iniciativa, al fortalecer la capacidad para manejar conflictos, tomar 
de decisiones y resolver desacuerdos, creando un ambiente propicio para el aprendizaje y el rendimiento académico.

\section{Referencias bibliográficas.}

1. Aguaded, M., \& Pantoja, M. (2015). Innovar desde un proyecto educativo de inteligencia emocional en primaria e infantil. Madrid, España: Dialnet.

2. Aguaded, M., \& Pantoja, M. (2015). Innovar desde un proyecto educativo de inteligencia en primaria e infantil. Tendencias pedagógicas. Madrid, España: Paidós.

3. Álvarez, M. (19 de Marzo de 2017). Misión posible: mejorar el bienestar de los orientadores a través de su inteligencia emocional. Asociación Española de Orientación y Psicopedagogía, 28(1), 15.

4. Aparicio, A., González, L., Portal, N., García, N., \& Lorente, L. (1 de Septiembre de 2016). Educar la inteligencia emocional de los estudiantes de Logofonoaudiología en la comunidad universitaria. Edumecentro, 15.

5. Bassedas, E. I. (2015). Intervención educativa y diagnóstico psicopedagógico. Barcelona, España: Paidós.

6. Beltrán, J. (2016). Claves psicológicas para la motivación y el rendimiento académico. En M. Acosta (Coord.), Creatividad, motivación y rendimiento académico. Málaga, España: Aljibe.

7. Bernal, A. (2013). La relación educativa, identidad y emociones. Madrid, España: Biblioteca Nueva.

8. Buitrago, D., \& Herrera, C. (2014). La inteligencia emocional y el tratamiento de las conductas disruptivas en el aula de clase. Ibagué, Colombia: Universidad del Tolima.

9. Buitrago, R. (2012). Contexto escolar e inteligencia emocional en instituciones educativas públicas del ámbito rural y urbano del departamento de Boyacá. Bogotá, Colombia: Universidad de Granada.

10. Cantó, J. D., \& Solbes, J. (2016). ¿Qué ciencias se enseñan y cómo se hace en las aulas de educación infantil? Segovia, España: Universidad de Valladolid.

11. Castro, C. (2014). Inteligencia emocional y violencia escolar. Bogotá, Colombia: Universidad Militar Nueva Granada.

12. Egido, M. (2016). La psicologización de la educación: implicaciones pedagógicas de la Inteligencia Emocional y Psicología Positiva. Educación XXI. Madrid, España: Centro de Estudios Superior Don Bosco.

13. Franco, L. (2014). Construcción de la democracia escolar y social desde los procesos de convivencia en las aulas. Caldas, Colombia: Universidad de Manizales. 
14. Fuentes, A. (1986). Procesos funcionales y eficacia de la escuela. Un modelo causal. Madrid, España: Universidad Complutense.

15. García, M., \& Giménez, S. (6 de Agosto de 2010). La inteligencia emocional y sus principales modelos. Espiral, Cuadernos el profesorado, 10.

16. González, T., Torres, L., \& Carreres, A. (22 de Marzo de 2017). Fortalezas y virtudes personales del profesorado y su relación con la eficacia docente. Revista INFAD de Psicología, 14.

17. Harris, A., \& Bennett, N. (2016). Eficacia Escolar y Mejoramiento Escolar. Perspectivas alternativas. Londres, Inglaterra: Continuum.

18. Hernández, P. (2015). Pensamiento, la Intuición y Emociones. Madrid, España: Tafor-Narcea.

19. Hose, C. (4 de Marzo de 2016). Juegos para enseñarles a los niños sobre las emociones. Universidad de Huelva, 12.

20. Jiménez, M. (2016). Inteligencias Múltiples en el rendimiento académico infantil. Guadalajara, México: Mc Graw Hill.

21. López, E. (3 de Diciembre de 2005). La educación emocional en la educación infantil. Revista Interuniversitaria de Formación del Profesorado, 8(3), 12.

22. López, E. (2015). La educación emocional en el aprendizaje infantil. Madrid, España: Paidós Ibérica.

23. López, P. (2015). Las Emociones en la Inteleigencia Emocional Infantil. California: Vergara.

24. Martín, M. (2013). Análisis de un Modelo Estructural de la Inteligencia Emocional y Motivación Auto determinada. Valencia, España : Universidad de Valencia.

25. Martineaud, S., \& Engelhart, D. (2014). Inteligencia emocional. Barcelona, España: Paidós Ibérica.

26. Mercadé, A. (20 de Febrero de 2016). Los 8 tipos de inteligencia según Howard Gardner. Editorial Académica Española, 15.

27. Miñaca, M., Hervas, M., \& Laprida, I. (13 de Julio de 2013). Análisis de programas relacionados con la Educación Emocional desde el modelo propuesto por Salovey \& Mayer. Universidad La Huelva, 18.

28. Moreno, C. (4 de Marzo de 2016). Estrategia de conducta. Pedagogía , 16.

29. Murillo, F. (2006). Murillo, F.J. (Coord.) (2006). Estudios sobre Eficacia Escolar en Iberoamérica. 15 buenas investigaciones. Bogotá, Colombia: Convenio Andrés Bello.

30. Navarro, R., Rodríguez, J., \& Eirín, R. (2016). Análisis de la satisfacción de las necesidades psicológicas básicas. Zaragoza, España: Coruña.

31. Pérez, L. (2013). De la evaluación a la intervención psicopedagógica. Madrid, España: Castellón. 
32. Roncancio, M., Camacho, N., Constanza, J., \& Vaca, P. (2017). Convivencia escolar y cotidianidad: una mirada desde la inteligencia emocional (Vol. 11). Barcelona, España: Paidós.

33. Sattler, J. (2014). Evaluación de la inteligencia infantil y habilidades especiales. Distrito Federal, México: El Manuel Moderno.

34. Silva, F. (2014). Evaluación psicológica en niños y adolescente. Madrid, España: Síntesis.

35. Silva, M. (2013). Habilidades emocionales en estudiantes de educación secundaria. Michoacán, México: Universidad Michoacana de San Nicolás de Hidalgo.

36. Tapia, A. (2014). Motivación y aprendizaje en el aula. Madrid, España: Santillana.

37. Touron, J. (2014). Factores del rendimiento académico en la Universidad. San Sebastián, España: Universidad de Navarro.

38. Valle, A., Cabanach, R., Rodríguez, S., Núñez, J., \& González, J. (2016). Metas académicas, estrategias cognitivas y estrategias de autorregulación del estudio. Asturias, Europa: Psicothema. 


\section{Para citar el artículo indexado.}

Andrade N., Andrade J., Andrade N., Peñafiel V. \& Andrade. A. (2018). La inteligencia emocional: una estrategia para mejorar el rendimiento académico, la comunicación y la pedagogía crítica.Revista electrónica Ciencia Digital 2(1), 360-374. Recuperado desde: http://www.cienciadigital.org/revistascienciadigital2/index.php/CienciaDigital/article/view/ $\underline{25 / 25}$

\section{LCiencia}

El artículo que se publica es de exclusiva responsabilidad de los autores y no necesariamente reflejan el pensamiento de la Revista Ciencia Digital.

El articulo queda en propiedad de la revista y, por tanto, su publicación parcial y/o total en otro medio tiene que ser autorizado por el director de la Revista Ciencia Digital.
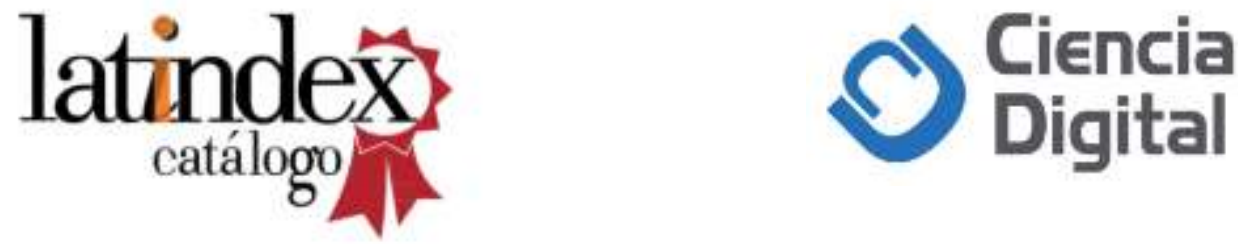